\title{
The cell surface protein Ag43 facilitates phage infection of Escherichia coli in the presence of bile salts and carbohydrates
}

\author{
Magdalena Gabig, ${ }^{1}$ Anna Herman-Antosiewicz, ${ }^{1}$ Marta Kwiatkowska, \\ Marcin Los, ${ }^{1}$ Mark S. Thomas ${ }^{2}$ and Grzegorz Weggrzyn ${ }^{1,3}$
}

\footnotetext{
1 Department of Molecular Biology, University of Gdansk, Kładki 24, 80-822 Gdansk, Poland

2 Division of Genomic Medicine, University of Sheffield Medical School, Beech Hill Road, Sheffield S10 2RX, UK

3 Institute of Oceanology, Polish Academy of Sciences, Św. Wojciecha 5, 81-347 Gdynia, Poland
}

\author{
Author for correspondence: Mark S. Thomas. Tel: +44 114271 2834. Fax: +44 1142739926 \\ e-mail:m.s.thomas@shef.ac.uk
}

\begin{abstract}
It was found that infection of Escherichia coli by bacteriophage $\lambda$ is inhibited in the presence of certain bile salts and carbohydrates when cells are in the 'OFF' state for production of the phase-variable cell surface protein antigen $\mathbf{4 3}$ (Ag43). The inhibition of phage growth was found to be due to a significant impairment in the process of phage adsorption. Expression of the gene encoding Ag43 (agn43) from a plasmid or inactivation of the oxyR gene (encoding an activator of genes important for defence against oxidative stress) suppressed this inhibition. A mutation, rpoA341, in the gene encoding the $\alpha$ subunit of RNA polymerase also facilitated phage adsorption in the presence of bile salts and carbohydrates. The rpoA341 mutation promoted efficient production of Ag43 in a genetic background that would otherwise be in the 'OFF' phase for expression of the agn43 gene. Analysis of a reporter gene fusion demonstrated that the promoter for the agn43 gene was more active in the rpoA341 mutant than in the otherwise isogenic rpoA+ strain. The combined inhibitory action of bile salts and carbohydrates on phage adsorption and the abolition of this inhibition by production of Ag43 was not restricted to $\lambda$, as a similar phenomenon was observed for the coliphages P1 and T4.
\end{abstract}

Keywords: bacteriophage infection, MacConkey agar, antigen 43, RNA polymerase $\alpha$ subunit, phase switching

\section{INTRODUCTION}

Escherichia coli lives in the intestinal tract where conditions may be far from those usually employed to culture this bacterium in the laboratory (LB medium), particularly in terms of nutrient availability, $\mathrm{pH}$ and oxygen tension. In mammals this bacterium is present in highest numbers in the anal proximal region of the lower bowel but it is also found in the small intestine (Drasar \& Barrow, 1985; Williams-Smith, 1965). This region of the gut contains the highest concentration of bile salts, compounds which are not found in LB medium. Bile salts are detergents made by the liver and excreted into the bile, and subsequently into the small intestine. In humans, the primary bile salts synthesized by the liver are cholate and chenodeoxycholate ( $41 \%$ and $39 \%$ of the total, respectively), which are subsequently conjugated to either glycine or taurine

Abbreviations: DOC, deoxycholate; PVP, polyvinylpyrrolidone; WSS Wytwórnia Surowic I Szczepionek. to form glycocholate and taurocholate, respectively (Elliott, 1985). In addition, deoxycholate (DOC; $15 \%$ of the total) is formed from cholate by the action of the resident intestinal microflora, which are also responsible for deconjugation of bile salts. The concentration of bile salts in the colon is greatly reduced, as the small intestine is also the site of absorption of $>90 \%$ of free and conjugated bile salts (Drasar \& Barrow, 1985; Elliott, 1985; Bernstein et al., 1999 and references therein). The ability of E. coli to resist the detergent action of bile salts is utilized for selective culture of this and other members of the Enterobacteriaceae from the intestinal tract or other sites of infection. The most commonly used selective medium is MacConkey agar, which contains DOC, or taurocholate and glycocholate, depending upon the manufacturer.

Antigen 43 (Ag43) is the major phase-variable protein in the outer membrane of E. coli and is present in excess of $5 \times 10^{4}$ molecules per cell (Owen, 1992). Ag43 is composed of two subunits, $\alpha$ and $\beta$, which are encoded by a single gene, agn43 (formerly called $f l u$ ) in E. coli K-12, 
and maturation requires removal of the $\mathrm{N}$-terminal signal peptide and proteolytic cleavage of the remaining polypeptide (Caffrey \& Owen, 1989; Henderson et al., 1997). The $43 \mathrm{kDa} \propto$ subunit is surface-expressed and is attached to the cell through an interaction between its C-terminal domain and the $43 \mathrm{kDa} \beta$ subunit, an integral outer-membrane protein (Caffrey \& Owen, 1989; Owen et al., 1987). Presentation of Ag43 on the cell surface is responsible for the so-called 'frizzy' or form 1 phenotype (Diderichsen, 1980; Henderson et al., 1997; Warne et al., 1990). This protein has also been reported to be a factor required for autoaggregation of E. coli cells (Henderson et al., 1997; Hasman et al., 1999, 2000), interspecies cell aggregation (Kjaergaard et al., 2000a, b) and cell-to-cell interactions within E. coli biofilms (Danese et al., 2000).

In this work we examined phage development in the presence of bile salt concentrations resembling those found in the intestinal tract (Pope et al., 1995 and references therein). Our results show that in the presence of carbohydrates, bile salts can strongly inhibit the ability of phage $\lambda$ and other coliphages to infect E. coli. This inhibition can be suppressed by the presence of Ag43 on the host cell surface, suggesting a potentially important role for this protein in interactions between E. coli and its phages in their natural environment.

\section{METHODS}

Bacteria, phages and plasmids. The Escherichia coli K-12 wild-type strain MG1655 (Jensen, 1993), WAM106 [F- araD139 $\Delta(\operatorname{argF}-l a c) U 169 \Delta($ his-gnd $)$ thi rpsL150 gltS flbB5301 relA1 deoC1 rbsR] and its rpoA341 derivative WAM105

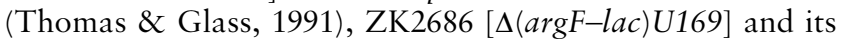
agn43::cat derivative ZK2692 (Danese et al., 2000), the $\triangle o x y R$ : : kan strain GSO9 (Zheng et al., 1999) and the dam13:: Tn9 strain GM2159 (a gift from M. Marinus, University of Massachusetts Medical School, MA, USA), were used. The rpoA341 mutation has been previously described and was isolated following chemical mutagenesis of E. coli CS71 (Giffard \& Booth, 1988; Zilberstein et al., 1980). Various alleles were transferred into different host genetic backgrounds by P1 transduction (Silhavy et al., 1984). Phages $\lambda$ cI857S7 (Goldberg \& Howe, 1969), גclb2, P1vir and T4 (from our collection) were employed. For cloning of the agn43 gene, a fragment of the MG1655 genome was amplified by PCR using the following primers: 5'-ATGGGATCCGGGACCACAGAGAGG-3' and 5'-CCGGAATTCGTTACTGTCTCTCTTGTC-3'. After amplification, the DNA fragment was digested with BamHI and EcoRI and cloned into the corresponding sites of pUC18 (Yanisch-Perron et al., 1985). The resultant plasmid was named pAG43g. For construction of the agn43lacZ transcriptional fusion, a DNA fragment encompassing the agn 43 promoter was amplified by PCR using the following primers: 5'-AGGATCCAGCAGGTATTCAAATGTCG-3' and 5'-CCGGAATTCGTTACTGTCTCTCTTGTC-3'. After amplification, the DNA fragment was digested with BamHI and EcoRI and cloned into the corresponding sites of pHG86 (Giladi et al., 1992), which harbours a copy of the lac $Z$ gene downstream of the multiple cloning site. The resultant plasmid was named pAG43p.

Culture media. Luria-Bertani (LB) medium and LB agar were as described by Sambrook et al. (1989). Various compounds
Table 1. Plaque formation by phage $\lambda$ on $E$. coli growing on MacConkey agar from different sources

Lactose was present at $1 \%$ in the MacConkey agar supplied by WSS and Oxoid, and was added to the same concentration in Difco media. All three sources of MacConkey agar contain peptones at a final concentration of $2 \%$. MacConkey agar from Difco and Oxoid contains conjugates of cholic acid $(0 \cdot 15 \%)$ whereas MacConkey agar from WSS contains sodium DOC $(0.09 \%)$. Sodium DOC was added to other media (where indicated) at a final concentration of $0 \cdot 09 \%$. Plaque formation was assessed by plating $\lambda c I b 2$ on E. coli MG1655.

\begin{tabular}{|lc|}
\hline MacConkey agar & Plaque formation* \\
\hline WSS & - \\
Oxoid & ++ \\
Difco & +++ \\
Difco + lactose & ++ \\
Difco + sodium DOC & ++ \\
Difco + lactose + sodium DOC & - \\
Oxoid + sodium DOC & - \\
\hline
\end{tabular}

$*+++$, Normal-sized plaques (diameter $>1.5 \mathrm{~mm}) ;++$, small plaques (diameter $0.5-1.5 \mathrm{~mm})$; - , absence of regular plaques (diameter $<0 \cdot 1 \mathrm{~mm}$, if any).

were added to the LB medium as indicated. Growth rates of all tested strains varied only slightly $(\leqslant 15 \%)$ in the presence of these compounds at the concentrations used (data not shown). Different sources of MacConkey agar were used: Difco Bacto MacConkey Agar Base, Oxoid MacConkey Agar and MacConkey Agar from WSS (Wytwórnia Surowic i Szczepionek). The compositions of these broths are broadly similar, but they differ in certain respects, most importantly in the nature of the included bile salts (for details see legend to Table 1).

Phage titration. Titration of bacteriophages was performed according to a standard procedure (Arber et al., 1983). Briefly, serial dilutions of a phage lysate were prepared in the test medium (containing supplements where indicated). Then, $2 \mu \mathrm{l}$ of each dilution was applied to a bacterial lawn, prepared by mixing of $0.2 \mathrm{ml}$ of an overnight culture of the indicator bacterial strain and $3 \mathrm{ml}$ of soft $(0.7 \%)$ nutrient agar (with or without supplements, as indicated), and the mixture was poured onto a plate containing the test agar medium. After allowing the drops to dry, the plates were incubated overnight at $37^{\circ} \mathrm{C}$. Alternatively, $0 \cdot 1 \mathrm{ml}$ of each phage dilution was mixed with $0.2 \mathrm{ml}$ of an overnight culture of the indicator bacterial strain and $3 \mathrm{ml}$ of soft nutrient agar (with or without supplements, as indicated) and poured onto a plate containing the test agar medium, followed by incubation as described above.

One-step growth experiments. Lytic development of bacteriophage $\lambda$ in E. coli cells was investigated by one-step growth experiments as described by Szalewska et al. (1994).

Measurement of the efficiency of phage adsorption. Bacteriophages were added to E. coli cells suspended in the appropriate medium to a m.o.i. of $0 \cdot 1$ and the mixture was incubated at $30^{\circ} \mathrm{C}$ (experiments performed at $37^{\circ} \mathrm{C}$ gave similar results although the kinetics of adsorption were different). Samples were withdrawn at the times indicated, centrifuged for $1 \mathrm{~min}$ in a microcentrifuge and the supernatant was titrated on the E. coli wild-type strain MG1655. The titre 
obtained at time zero (a sample withdrawn immediately after addition of bacteriophages to the cell suspension) was considered to correspond to $100 \%$ unadsorbed phages and other values were calculated relative to this value.

Bacterial autoaggregation assay. Autoaggregation of bacterial cells was tested according to Hasman et al. (1999). Briefly, overnight cultures of tested strains were adjusted to approximately the same $\mathrm{OD}_{575}$ (about $1 \cdot 2$ ) by dilution with the same medium and $15 \mathrm{ml}$ each culture was placed in a sterile $20 \mathrm{ml}$ tube and vigorously agitated for $10 \mathrm{~s} .1 \mathrm{ml}$ samples were withdrawn from approximately $1 \mathrm{~cm}$ below the meniscus at the indicated times and the $\mathrm{OD}_{575}$ was measured.

Isolation and analysis of membrane proteins. Cell and protein fractionation by sucrose density gradient ultracentrifugation was performed as described by Kucharczyk et al. (1991) and Kȩdzierska et al. (1999). The fractions of outer- and inner membrane proteins were collected separately and subjected to SDS-PAGE.

Protein sequencing. After transfer of the material from selected protein bands obtained after SDS-PAGE to an Immobilon P membrane (Bio-Rad), automatic microsequencing was performed at the Institute of Molecular Biology, BioCenter, Jagiellonian University (Cracow, Poland).

Measurement of $\boldsymbol{\beta}$-galactosidase activity. Activity of $\beta$ galactosidase in cells harbouring a fusion of the agn43 promoter to the lacZ gene was measured according to Miller (1972).

\section{RESULTS}

A combination of certain bile salts and carbohydrates inhibits the formation of $\lambda$ plaques

We found that bacteriophage $\lambda$ cannot form regular plaques on wild-type E. coli growing on lactose/ MacConkey Agar produced by WSS. [Detailed inspection of the plates under a magnifying glass revealed the formation of extremely small plaques (even smaller than plaques normally described as 'pin-point', i.e. $<0 \cdot 1$ $\mathrm{mm}$ ), which were present at a number corresponding to an e.o.p. close to 1 (relative to the e.o.p. on LB plates). Nevertheless, as these plaques were so tiny as to be invisible to the naked eye, for simplicity such a phenotype is described here as 'no plaque formation' (or '-').] However, normal sized $\lambda$ plaques were obtained on wild-type E. coli growing on Difco or Oxoid lactose/ MacConkey agar (Table 1). Although the composition of the MacConkey media obtained from the different sources is similar, certain components differ, particularly the nature of the bile salts. MacConkey agar obtained from WSS contains DOC $(0.09 \%$, w/v) whereas the other suppliers incorporate a mixture of cholic acid conjugated with the amino acids glycine and taurine $(0 \cdot 15 \%, \mathrm{w} / \mathrm{v})$. Addition of DOC to the Difco and Oxoid media inhibits plaque formation on E. coli, but only in the presence of lactose (Table 1).

To determine whether this phenomenon was dependent only on the presence of DOC and lactose, we added the individual MacConkey components separately and in various combinations to LB agar, and titrated bacteriophage $\lambda$ on E. coli lawns grown on each composite
Table 2. Effects of different bile salts, detergents and other related compounds on $\lambda$ plaque formation in the presence and absence of lactose

Bile salts and other reagents were added to LB agar at a final concentration of $0.09 \%$. Plaque formation was assessed by plating $\lambda c I b 2$ on E. coli MG1655. Lactose (where present) was added at a final concentration of $1 \%$.

\begin{tabular}{|lcc|}
\hline Bile salt/detergent & \multicolumn{2}{c|}{ Plaque formation* } \\
\cline { 2 - 3 } & - lactose & + lactose \\
\hline None & & \\
DOC & +++ & ++ \\
Cholate & ++ & - \\
Chenodeoxycholate & ++ & - \\
Lithocholic acid & ++ & - \\
Cholanic acid & ++ & - \\
Glycocholate & +++ & ++ \\
Taurocholate & +++ & ++ \\
Glycodeoxycholate & +++ & ++ \\
Taurodeoxycholate & +++ & ++ \\
CHAPS & +++ & ++ \\
Cholesterol & +++ & ++ \\
Sarkosyl & ++ & ++ \\
SDS & +++ & + \\
Triton X-100 & +++ & + \\
Brij 58 & +++ & ++ \\
PEG 6000 & +++ & ++ \\
PVP & +++ & + \\
\hline
\end{tabular}

$*+++$, Normal-sized plaques (diameter $>1.5 \mathrm{~mm}) ;++$, small plaques (diameter $0.5-1.5 \mathrm{~mm})$; + , very small plaques (diameter $0 \cdot 1-0 \cdot 5 \mathrm{~mm}) ;-$, absence of regular plaques (diameter $<0 \cdot 1 \mathrm{~mm}$, if any).

medium. In no case was formation of plaques inhibited when components of MacConkey agar were tested singly, although addition of lactose or DOC resulted in the production of smaller plaques. Only when lactose was present together with DOC was $\lambda$ plaque formation abolished. The nature of the carbohydrate (whether pentose or hexose, or mono- or disaccharide) present in the medium in combination with DOC does not appear to be important for the inhibition of phage $\lambda$ development. Furthermore, metabolism of the carbohydrate does not play a role in the inhibitory process, as plaque formation on a $\mathrm{lac}^{-}$tester strain, WAM106, was also found to be inhibited by the presence of DOC and lactose together (data not shown). To determine whether the inhibitory effect is specific for carbohydrates or also occurs in the presence of other related carbon sources, we tested the effects of the polyalcohols glycerol and mannitol in combination with DOC on plaque formation by phage $\lambda$. We observed only a slight reduction in plaque size in the presence of these components relative to the plaque size formed in the presence of DOC alone (data not shown). Pyruvate together with DOC caused some reduction in plaque 


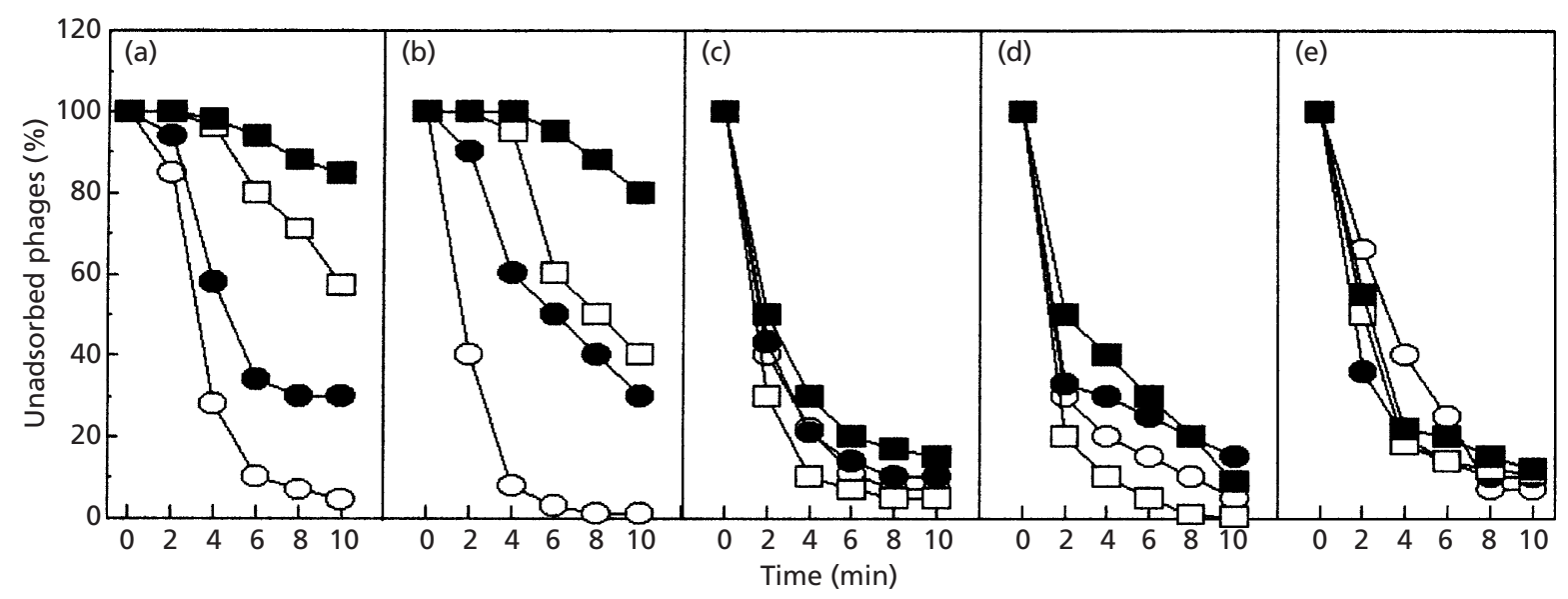

Fig. 1. Effect of medium composition and agn43 expression on adsorption of bacteriophage $\lambda$ to $E$. coli. Adsorption of phage $\lambda \mathrm{clb} 2$ on E. coli rpoA ${ }^{+}$strains MG1655 and WAM106 ( $\mathrm{a}$ and b, respectively), the $E$. coli rpoA341 mutant WAM105 (c) and strains WAM106 and WAM105 harbouring the agn43-overexpressing plasmid, pAG43g (d and e, respectively), was measured in LB medium (white circles), LB supplemented with $1 \%$ lactose (black circles), LB supplemented with $0.09 \%$ sodium DOC (white squares) and LB supplemented with $1 \%$ lactose and $0.09 \%$ sodium DOC (black squares).

size but not as much as pentoses, hexoses and disaccharides (data not shown). Thus, it seems that the strong inhibitory effect on plaque formation is specific to carbohydrates.

We tested whether other bile salts and bile acids, acting alone or together with carbohydrates, are able to inhibit phage development in LB medium. Addition of unconjugated bile salts containing one, two or three hydroxyl groups (lithocholate, chenodeoxycholate and cholate, respectively) had similar effects to those exerted by DOC (two hydoxyl groups), whereas cholanic acid, a bile acid metabolite devoid of hydroxyl groups, was only slightly inhibitory for plaque formation (Table 2). The conjugated forms of cholate and DOC also had little effect on $\lambda$ plaque formation (Table 2). We also examined the effect of the structurally related (i.e. steroidal) detergent CHAPS and the non-detergent bile salt precursor cholesterol, as well as other unrelated detergents, both ionic (Sarkosyl, SDS) and non-ionic (Brij 58, Triton X-100), on plaque formation. While addition of all these compounds to LB medium did result in a reduction in plaque size in the presence of added carbohydrate (particularly Sarkosyl and Triton X-100) none of them completely inhibited plaque formation (Table 2). Other compounds that increase the viscosity of the medium, such as PEG and PVP (polyvinylpyrrolidone), only exerted slight inhibitory effects on plaque formation in the presence of carbohydrate (Table 2).

The threshold (minimal inhibitory) concentrations of bile salts and carbohydrates for prevention of $\lambda$ plaque formation were also determined. Different combinations of DOC and lactose were used in LB plates and formation of $\lambda$ plaques was monitored. We estimated that the threshold concentrations of DOC and lactose that completely block formation of normal $\lambda$ plaques are $0.04 \%$ and $0.4 \%$, respectively (data not shown). These concentrations were inhibitory only when the second inhibitory compound (either lactose or DOC, respectively) was also present in the medium at the inhibitory concentration.

\section{Bile salts in combination with carbohydrates impair phage $\lambda$ adsorption}

To identify the step that is inhibited by the combined presence of bile salts and carbohydrates, phage growth was monitored in one-step growth experiments where the phage and E. coli host were exposed to DOC and lactose either before or following phage adsorption. No significant influence of these compounds on phage growth (as judged by measurement of phage burst size) was observed when added after phage adsorption (data not shown). When we tested the efficiency of $\lambda$ adsorption in the presence of DOC or lactose we found that phage adsorption was somewhat less effective in the presence of either of these components $(\sim 60 \%$ and $30 \%$ of phages remained unadsorbed in the presence of DOC or lactose alone, respectively, in comparison to less than $10 \%$ phages remaining unadsorbed in the absence of these compounds). However, adsorption was severely impaired by the combined presence of DOC and lactose ( $\sim 90 \%$ phages remained unadsorbed) (Fig. 1a). Other carbohydrates in combination with DOC gave rise to similar results (data not shown). Thus, we conclude that the inhibition of phage $\lambda$ development by the combined action of bile salts and carbohydrates is mainly due to impairment of phage adsorption on the host cells.

\section{Inhibition of phage adsorption does not require de novo protein synthesis}

To distinguish between the possibility that the combined action of carbohydrates and bile salts on phage infection is due to a physical or chemical action on the cell 
Table 3. Effect of DOC and lactose on plaque formation by phages $\lambda, \mathrm{P} 1$ and T4 on $E$. coli $r p o A^{+}$and $r p o A 341$ hosts in the presence and absence of Ag43 overproduction

Lactose was added to LB agar to a final concentration of $1 \%$ and sodium DOC was added to a final concentration of $0.09 \%$. Plaque formation was assessed by plating $\lambda c I b 2$, P1vir and T4 on E. coli WAM106 $\left(r p o A^{+}\right)$and WAM105 (rpoA341) hosts. Plasmid pAG43g bears a copy of the agn43 gene under control of the lacUV5 promoter.

\begin{tabular}{|c|c|c|c|c|c|}
\hline \multirow[t]{2}{*}{ Phage } & \multirow{2}{*}{$\begin{array}{l}\text { Additional } \\
\text { components }\end{array}$} & \multicolumn{4}{|c|}{ Phage plaque formation on strain...* } \\
\hline & & $\operatorname{rpo} A^{+}$ & $r p o A^{+} / \mathrm{pAG} 43 \mathrm{~g}$ & rpo $A 341$ & rpoA341/pAG43g \\
\hline$\lambda$ & $\begin{array}{l}\text { None } \\
\text { DOC } \\
\text { Lactose } \\
\text { Lactose + } \\
\text { DOC }\end{array}$ & $\begin{array}{c}+++ \\
++ \\
++ \\
-\end{array}$ & $\begin{array}{l}+++ \\
+++ \\
+++ \\
+++\end{array}$ & $\begin{array}{l}+++ \\
+++ \\
+++ \\
+++\end{array}$ & $\begin{array}{l}+++ \\
+++ \\
+++ \\
+++\end{array}$ \\
\hline P1 & $\begin{array}{l}\text { None } \\
\text { DOC } \\
\text { Lactose } \\
\text { Lactose + } \\
\text { DOC }\end{array}$ & $\begin{array}{c}+++ \\
++ \\
++ \\
-\end{array}$ & $\begin{array}{c}+++ \\
++ \\
++ \\
+\end{array}$ & $\begin{array}{c}+++ \\
++ \\
++ \\
+\end{array}$ & $\begin{array}{c}+++ \\
++ \\
++ \\
+\end{array}$ \\
\hline $\mathrm{T} 4$ & $\begin{array}{l}\text { None } \\
\text { DOC } \\
\text { Lactose } \\
\text { Lactose + } \\
\text { DOC }\end{array}$ & $\begin{array}{c}+++ \\
++ \\
++ \\
-\end{array}$ & $\begin{array}{c}+++ \\
+++ \\
+++ \\
++\end{array}$ & $\begin{array}{c}+++ \\
+++ \\
+++ \\
++\end{array}$ & $\begin{array}{l}+++ \\
+++ \\
+++ \\
+++\end{array}$ \\
\hline
\end{tabular}

$*+++$, Normal-sized plaques (diameter $>1.5 \mathrm{~mm}) ;++$, small plaques (diameter $0.5-1.5 \mathrm{~mm}$ ); + , very small plaques (diameter $0 \cdot 1-0.5 \mathrm{~mm}$ ); - , absence of regular plaques (diameter $<0.1 \mathrm{~mm}$, if any).

envelope, or the result of an alteration in expression of certain bacterial genes in response to these agents, the effect of DOC and lactose on the efficiency of phage adsorption was measured in the presence of the transcription inhibitor rifampicin $\left(25 \mu \mathrm{g} \mathrm{ml}^{-1}\right)$ or the protein synthesis inhibitor chloramphenicol $\left(35 \mu \mathrm{g} \mathrm{ml}^{-1}\right)$. The results obtained were very similar to those observed in the absence of these antibiotics (data not shown), indicating that the mechanism of inhibition does not require gene transcription or protein synthesis subsequent to the addition of carbohydrates and bile salts.

\section{The rpoA341 mutation suppresses the inhibition of $\lambda$ phage adsorption and plaque formation in the presence of bile salts and carbohydrates}

The $r p o A$ gene encodes the $\alpha$ subunit of RNA polymerase. This subunit plays a role in activator- and UP element-dependent transcription at many promoters. We found that phage $\lambda$ forms normal plaques on lawns of a strain (WAM105) harbouring a mutant rpoA allele, rpoA341, in the presence of DOC and lactose, whereas an otherwise isogenic rpo $A^{+}$strain (WAM106) behaved in an identical fashion to the wild-type E. coli strain MG1655 (Table 3). Indeed, with all the combinations of bile salts/detergents and carbohydrates described above, the ability of the WAM106 strain $\left(r p o A^{+}\right)$to support plaque formation was the same as that of
MG1655, whereas $\lambda$ formed normal plaques on lawns of the rpoA341 mutant in the presence of all of the combinations tested (data not shown). We also found that whereas phage $\lambda$ adsorbs with low efficiency to the $r p o A^{+}$host (WAM106) in the presence of DOC and lactose, these agents have little inhibitory effect on $\lambda$ adsorption to the rpoA341 mutant (Fig. 1b, c).

\section{The level of Ag43 is increased in the rpoA341 mutant relative to the $r p o A^{+}$strain}

The rpoA341 mutation impairs transcription of a number of positively regulated genes. As the inhibitory effect of DOC and lactose on plaque formation appears to be exerted at the level of phage adsorption, we compared profiles of outer and inner membrane proteins of the otherwise isogenic rpoA341 and rpo $A^{+}$strains grown in the absence of bile salts. We found differences between the amounts of several inner- and outer membrane proteins in these strains (results not shown). However, the most striking difference was the presence of a considerable increase in the abundance (almost 10fold, as estimated by densitometric analysis of bands on the gel) of an outer membrane protein of molecular mass $\sim 45 \mathrm{kDa}$ in the rpoA341 strain relative to the rpo $A^{+}$ parent. To identify this protein, the appropriate band was cut from the gel, transferred to an Immobilon P membrane and the $\mathrm{N}$ terminus of the polypeptide was 
Table 4. Effect of DOC and lactose on agn 43 promoter activity in E. coli rpoA $A^{+}$(WAM106) and rpoA341 (WAM105) hosts

Lactose was added to a final concentration of $1 \%$, and sodium DOC was added to final concentration of $0.09 \%$.

\begin{tabular}{|c|c|c|}
\hline \multirow[t]{2}{*}{ Medium } & \multicolumn{2}{|c|}{$\begin{array}{c}\beta \text {-Galactosidase activity } \\
(\text { Miller } U)^{*}\end{array}$} \\
\hline & $\operatorname{rpo} A^{+}$ & rpo $A 341$ \\
\hline $\mathrm{LB}$ & $4830 \pm 670$ & $9550 \pm 150$ \\
\hline $\mathrm{LB}+$ sodium DOC & $2890 \pm 430$ & $8160 \pm 910$ \\
\hline $\mathrm{LB}+$ lactose & $2900 \pm 570$ & $6960 \pm 900$ \\
\hline $\mathrm{LB}+$ lactose + sodium DOC & $2870 \pm 420$ & $7870 \pm 100$ \\
\hline
\end{tabular}

* Mean values from three experiments \pm standard deviations are presented.

sequenced. This sequence, ADIVVHPGETV, was found to be $100 \%$ identical to the processed $\mathrm{N}$ terminus of the $\alpha$ subunit of a cell surface protein known as Ag43. No other E. coli protein revealed $100 \%$ identity with this sequence, as analysed by computer-mediated search (WU-BLASTP software). Therefore, we conclude that increased production of Ag43 occurs in the presence of the rpoA341 mutation.

\section{Activity of the agn43 promoter is stimulated by the rpoA341 mutation}

To test whether the increased abundance of $\mathrm{Ag} 43$ in the rpoA341 mutant was due to an increase in the efficiency of transcription of the gene encoding this protein, agn 43 (formerly known as $f l u$ ), we constructed a transcriptional fusion consisting of the agn43 promoter and the lac $Z$ reporter gene. We found that the agn 43 promoter is active in the rpo $A^{+}$strain under all conditions tested, although it is depressed by about $40 \%$ in the presence of lactose alone, DOC alone, or lactose and DOC together (Table 4). However, the $\beta$-galactosidase activity in the rpoA341 mutant harbouring this fusion is 2-3-fold higher than that in the $r p o A^{+}$strain, and is less strongly affected by the presence of DOC and/or lactose in the medium (Table 4). As the fusion was present on a plasmid, we measured the plasmid copy number in both strains and found no significant difference (data not shown). Therefore, the increased abundance of Ag43 in the rpoA341 mutant is due, at least in part, to increased transcription of agn 43 .

\section{Effect of the rpoA341 mutation on the Ag43 phenotype}

Production of Ag43 is subject to reversible phase variation, with rates of transition from the $\mathrm{Ag} 43^{+}$ (the ' $\mathrm{ON}$ ' phase) to $\mathrm{Ag} 43^{-}$state (the 'OFF' phase) being $\sim 2 \cdot 2 \times 10^{-3}$, and from $\mathrm{Ag} 43^{-}$to $\mathrm{Ag} 43^{+}$being

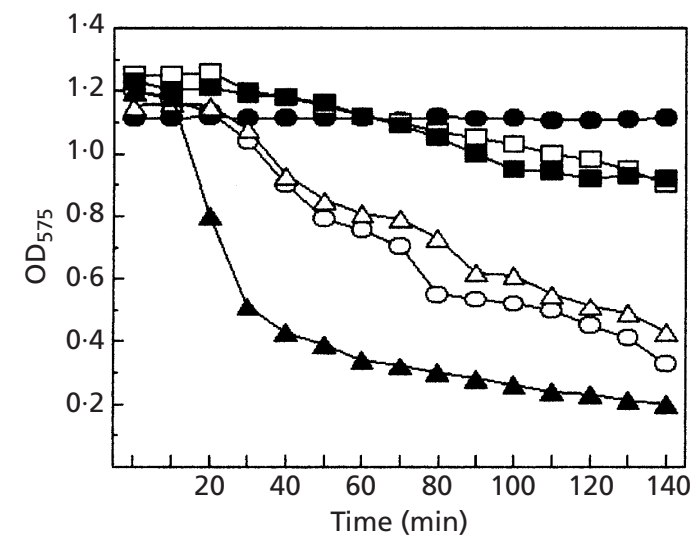

Fig. 2. Autoaggregation of bacterial cells. Cultures of ZK2686 (Ag43 ${ }^{+}$, white circles), ZK2692 (agn43::cat, black circles), MG1655 (Ag43-, white squares), WAM106 (Ag43-', black squares), WAM105 (rpoA341 derivative of WAM106, white triangles) and WAM106 bearing the $\triangle$ oxyR::kan allele (black triangles) were kept stationary in a tube, and $\mathrm{OD}_{575}$ of samples withdrawn approximately $1 \mathrm{~cm}$ below the meniscus was measured at the times indicated.

$\sim 1 \cdot 0 \times 10^{-3}$ (Caffrey \& Owen, 1989; Henderson et al., 1997). Therefore, we tested the Ag43 status of the strains employed in our experiments using an autoaggregation assay (Hasman et al., 1999). Bacteria producing Ag43 form cellular aggregates and sediment efficiently in a stationary culture, whereas sedimentation of $\mathrm{Ag} 43^{-}$ strains is negligible. As reference strains we used a pair of $a g n 43^{+}$and agn43::cat bacteria, ZK2686 and ZK2692, respectively (Danese et al., 2000). As expected, ZK2686 $\left(\operatorname{agn} 43^{+}\right)$bacteria sedimented efficiently, whereas autoaggregation of the agn43::cat mutant (ZK2692) was impaired (Fig. 2). We found that autoaggregation of MG1655 and WAM106 bacteria is also inefficient, although, unlike the agn43::cat mutant (ZK2692), some sedimentation of these strains was observed at later stages of the assay (Fig. 2). The rpoA341 mutant, WAM105, sedimented as efficiently as the Ag43+ control strain, ZK2686 (Fig. 2). To confirm that the difference in the autoaggregation behaviour of WAM105 and WAM106 was not due to the chance isolation of 'locked ON' and 'locked OFF' strains, respectively, during preparation of our stocks, we transduced the rpoA341 allele into WAM106. WAM106 rpoA341 transductants were found to autoaggregate as efficiently as WAM105.

Phase switching of Ag43 is a result of the action of Dam methylase, a positive regulator of agn43, and OxyR, which is a negative regulator of agn43 (Henderson et al., 1997; Henderson \& Owen, 1999; Haagmans \& van der Woude, 2000). oxyR mutants are locked in the ' $O N$ ' state for agn43 expression. Introduction of the $\Delta o x y R:$ :kan mutation into the WAM106 strain also resulted in very efficient autoaggregation of bacterial cells (Fig. 2). These results indicate that the MG1655 and WAM106 isolates used by us are mostly in the 'OFF' state for agn43 expression, with perhaps a small 
Table 5. Effect of agn43 gene function on plaque formation by phage $\lambda$ in the presence of DOC and lactose

Plasmid pAG43g bears the agn43 gene under control of the lacUV5 promoter. Lactose was added to a final concentration of $1 \%$ and sodium DOC was added to a final concentration of $0.09 \%$ to LB medium. Plaque formation was assessed by plating $\lambda c l b 2$ on each strain.

\begin{tabular}{|c|c|}
\hline Host & $\begin{array}{c}\text { Plaque } \\
\text { formation* }\end{array}$ \\
\hline WAM106 (rpo $\left.A^{+} \mathrm{Ag}^{4} 3^{-}\right)$ & - \\
\hline WAM106 agn43::cat $\left(r p o A^{+} A g 43^{-}\right)$ & - \\
\hline WAM105 (rpoA341 Ag43) & +++ \\
\hline WAM105 agn43::cat (rpoA341 Ag43-) & - \\
\hline ZK2686 $\left(\operatorname{agn} 43^{+}\right)$ & +++ \\
\hline ZK2686 $\left(\operatorname{agn} 43^{+}\right) / \mathrm{pAG} 43 \mathrm{~g}$ & +++ \\
\hline ZK2692 (agn43::cat) & - \\
\hline ZK2692 (agn43::cat)/pAG43g & +++ \\
\hline
\end{tabular}

$*+++$, Normal-sized plaques (diameter $>1.5 \mathrm{~mm}$ ) ; -, absence of regular plaques (diameter $<0 \cdot 1 \mathrm{~mm}$, if any).

proportion of the cell population locked in the ' $\mathrm{ON}$ ' state, whereas WAM105 is mainly in the ' $\mathrm{ON}$ ' state. Furthermore, the rpoA341 allele is specifically responsible for locking strains in the ' $O N$ ' phase for agn43 expression.

\section{The rpoA341 mutation increases the sensitivity of $E$. coli to hydrogen peroxide}

As OxyR is a negative regulator of agn 43 expression, the rpoA341 mutation could exert its stimulatory effect on agn43 transcription indirectly, through impairing expression of $o x y R$. As oxyR mutants are sensitive to hydrogen peroxide, we tested the possibility that strains bearing the mutant RNA polymerase are sensitive to hydrogen peroxide. Bacteria containing either the rpoA341 allele or both the rpoA341 and $\Delta o x y R:$ :kan alleles together were found to be at least as sensitive to hydrogen peroxide as the $\Delta o x y R:$ : kan mutant (data not shown). This observation is consistent with the possibility that the rpoA341 mutation results in impairment of $o x y R$ expression.

\section{Ag43 suppresses the inhibitory effect of bile salts and carbohydrates on adsorption and plaque formation by phage $\lambda$}

To test whether the ability of phage $\lambda$ to efficiently adsorb to a strain carrying the rpoA341 mutation in the presence of bile salts and carbohydrate is specifically due to the increased production of $\mathrm{Ag} 43$ in this strain, we constructed an rpoA341 agn43::cat double mutant. This strain was found to be unable to support phage adsorption and the formation of normal plaques by $\lambda$ in the presence of DOC and lactose (Table 5). Therefore, we investigated the effect of $\mathrm{Ag} 43$ overproduction on the

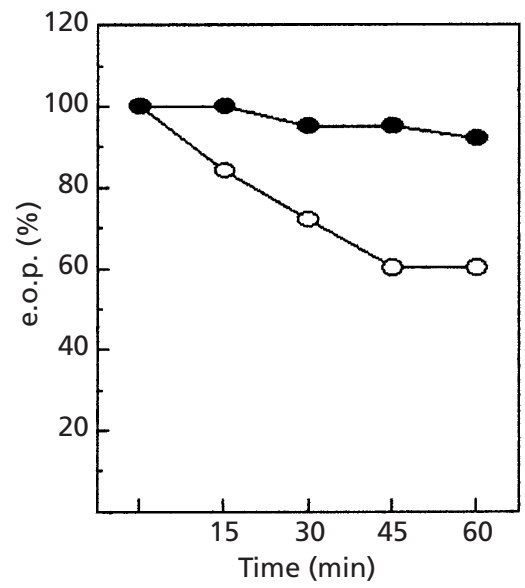

Fig. 3. Effect of preincubation of $\lambda$ phage with fractions of outer membrane proteins isolated from strains ZK2686 ( $\mathrm{Ag}^{+} 3^{+}$, white circles) and ZK2692 (agn43::cat, black circles) on the e.o.p. on the indicator strain (MG1655). A phage lysate $(0.3 \mathrm{ml})$ containing $4 \times 10^{7}$ p.f.u. $\mathrm{ml}^{-1}$ was incubated with outer membrane protein fractions ( $27 \mathrm{ng}$ protein per sample) for the lengths of time indicated, and then titrated on agar plates. e.o.p. estimated at time 0 was considered to be $100 \%$.

efficiency of phage $\lambda$ adsorption to the $r p o A^{+}$strain. As expected, we found that in WAM106 bacteria in which Ag43 production was directed from a multicopy plasmid (pAG43g) the cells autoaggregated efficiently. Moreover, phage $\lambda$ adsorption on this strain was efficient irrespective of the presence of DOC and/or lactose (Fig. $1 \mathrm{~d}, \mathrm{e})$. The improved adsorption of phage $\lambda$ on $\mathrm{Ag} 43-$ overproducing $r p o A^{+}$cells in the presence of DOC and lactose also resulted in restoration of the ability of this strain to support normal plaque formation under these conditions (Table 3). Similarly, we found that in the presence of DOC and lactose, $\lambda$ forms normal plaques on the reference $a g n 43^{+}$host (ZK2686), but not on the agn43:: cat mutant (ZK2692). The inability of phage $\lambda$ to form plaques on ZK2692 was suppressed by expression of agn43 from a plasmid (Table 5). These results strongly suggest that the ability of the rpoA341 host to support phage development in the presence of bile salts and carbohydrates is due to a specific stimulatory effect of this mutation on agn 43 gene expression.

\section{Protein fractions containing Ag43 sequester phage and lower the number of p.f.u.}

To test whether Ag43 may physically attach to phage $\lambda$, we preincubated phage lysates with outer membrane protein fractions isolated from agn43 ${ }^{+}$(ZK2686) and agn43::cat (ZK2692) strains. Then phages were titrated on the indicator strain (MG1655). We found that the titre of $\lambda$ decreased significantly after incubation with the outer membrane protein fraction isolated from the agn $43^{+}$bacteria, in contrast to phage incubated with outer membrane protein fractions obtained from the agn 43:: cat mutant bacteria (Fig. 3). Therefore, it seems that $\mathrm{Ag} 43$ may sequester phage $\lambda$ when added separately 
to a phage lysate, and under these experimental conditions it may act as a competitor for the binding of phages.

\section{Ag43 affects phage $\lambda$ adsorption only in the presence of bile salts and carbohydrates}

We asked whether production of Ag43 is physiologically important for $\lambda$ adsorption under all growth conditions or only in the presence of bile salts and carbohydrates. We found that phage $\lambda$ forms normal plaques on the agn $43:$ : cat mutant and on a dam :: cat mutant (in which agn43 expression is locked in the 'OFF' phase) on LB agar plates (data not shown). As expected, formation of $\lambda$ plaques on these hosts was impaired in the presence of DOC and lactose (Table 3 and data not shown). These results clearly demonstrate that expression of the agn 43 gene is important for phage $\lambda$ infection only in the presence of bile salts and carbohydrates.

\section{The inhibitory effect of carbohydrates and bile salts on phage growth is not restricted to bacteriophage $\lambda$}

To examine whether the combined action of bile salts and carbohydrates on phage infection is specific only for $\lambda$ we examined plaque formation by phages $\mathrm{P} 1$ and T4 in the presence of lactose and DOC. We found that the presence of these compounds affects plaque formation by both phages in a similar manner to that observed for $\lambda$, i.e. plaque formation by $\mathrm{P} 1$ and $\mathrm{T} 4$ was inhibited by the presence of lactose and DOC (Table 3). Moreover, in both cases phage adsorption was shown to be impaired by the presence of lactose and DOC (data not shown). Furthermore, this inhibition was abolished by either the presence of the rpoA341 mutation or expression of the agn43 gene from a multicopy plasmid, although the stimulatory effects of agn 43 expression on plaque formation by phage P1 were less pronounced than for $\lambda$ and T4 (Table 3). Since plaque formation by $\lambda, \mathrm{P} 1$ and T4 phages in the presence of DOC and lactose was restored by the same genetic factors, yet their target receptors differ, it seems that the inhibition of phage development is due to a general physical or chemical effect on phage adsorption rather than directed at a specific phage receptor.

\section{Bile salts together with carbohydrates may sequester divalent cations and prevent effective phage adsorption but can be compensated for by the presence of Ag43 on the cell surface}

Many phages require the presence of divalent cations for stability and to neutralize the negative charge of the bacterial cell surface to allow efficient adsorption to their hosts (Casjens \& Hendrix, 1988; Hancock, 1997). Under conditions of relatively low cation concentration (we do not supplement LB media with divalent cations) any sequestration of cations, or exclusion of cations from the bacterial cell surface, by the combined action of bile salts and carbohydrates could explain the observed inhibition of phage adsorption. In support of this idea, we found that the inhibitory effect of bile salts and carbohydrates on plaque formation by phages $\lambda$ and T4 on MG1655 or WAM106 can be suppressed by addition of $\mathrm{Mg}^{2+}(20 \mathrm{mM})$ to the medium, whereas addition of $\mathrm{Ca}^{2+}(20 \mathrm{mM})$ is required to suppress the effect on plaque formation by P1 (data not shown).

\section{DISCUSSION}

\section{A mechanism for inhibition of phage development by bile salts and carbohydrates}

We have found that several bacteriophages cannot form plaques on a wild-type E. coli host in the presence of certain bile salts together with a variety of different carbohydrates. Our results strongly suggest that this inhibition is due to impairment of phage adsorption. Both of these classes of compound impair phage adsorption and plaque formation to some degree when present alone. However, increasing the concentration of either DOC or lactose further, when either is present alone, did not result in a stronger inhibitory effect on adsorption (data not shown). This observation suggests that these compounds are acting synergistically to inhibit phage adsorption. Metabolism of the carbohydrate is not required for the negative effect on phage development. The reduction of phage adsorption was not caused solely by increased viscosity of the medium, as PVP did not significantly affect $\lambda$ plaque formation. The inhibition of phage adsorption by certain bile salts is not due solely to their action as general detergents, as other structurally unrelated detergents were not able to completely abolish plaque formation. Indeed, apart from Triton X-100 and Sarkosyl, the effects of the other detergents tested were not significant. Effects on cell surface hydrophobicity are also unlikely to explain this phenomenon as it has been shown that DOC does not increase cell surface hydrophobicity (Pope et al., 1995).

Few conclusions can be drawn regarding the structural requirements of the inhibitory detergents. Like all bile acids, the inhibitory bile acids (cholate, DOC, chenodeoxycholate and lithocholate) are steroid-based molecules, possessing between one and three hydroxyl groups in addition to the alkyl side chain carboxyl group. The hydroxyl groups attached to the steroid moiety mean that the molecule has polar and non-polar faces. Although dihydroxy bile salts (DOC, chenodeoxycholate) are more effective than trihydroxy bile salts (cholate) in membrane solubilization and dissociation of protein-protein interactions, we observed no relationship between this property and their effect on $\lambda$ plaque formation. Cholanic acid, which is not inhibitory for plaque formation, is identical to these bile acids except that it does not possess hydroxyl groups. On the other hand, the bile acid precursor cholesterol, which is also not inhibitory for plaque formation, does possess a hydroxyl group attached to the steroid moiety but the aliphatic side chain is larger and lacks a carboxyl group. Interestingly, amidation of the single carboxyl group of cholic acid or DOC with an amino acid, despite resulting in replacement of the original bile acid carboxyl group 
with an alternative free carboxyl or sulfonyl group (e.g. glycodeoxycholate, taurodeoxycholate or CHAPS), diminishes the potency of these compounds.

As infection of E. coli by other phages that employ different receptors for attachment to the host bacterial cell surface are similarly prone to interference by bile salts and carbohydrates, the mechanism of inhibition of phage adsorption is unlikely to involve specific effects on their receptors. The possibility of a direct effect of bile salts and carbohydrates on the phages themselves is also unlikely because we found that overproduction of a bacterial protein, Ag43, serves to restore efficient phage adsorption and plaque formation in the presence of bile salts and carbohydrates. Our results suggest that bile salts together with carbohydrates may sequester cations or deplete them from the cell surface and thereby prevent effective phage adsorption. This inhibition can be circumvented by the presence of $\mathrm{Ag} 43$ on the cell surface. Since the $\alpha$ subunit of $\mathrm{Ag} 43$ extends beyond the LPS O antigen (Henderson et al., 1997) and can function as an adhesin mediating autoaggregation and biofilm formation (Diderichsen, 1980; Danese et al., 2000), this protein could serve as an alternative initial site of interaction of the phage with the host under limiting concentrations of divalent cations. The possibility that phage adsorption is somehow facilitated by $\mathrm{Ag} 43$ mediated aggregation of bacteria, rather than due to an interaction with $\mathrm{Ag} 43$ is unlikely, as $\mathrm{Ag} 43$-induced suppression also occurs in solid medium.

\section{Role of the RNA polymerase $\alpha$ subunit in regulation of agn43 expression}

Phase variation of $\mathrm{Ag} 43$ is regulated at the transcriptional level and requires the Dam methylase and the regulatory protein OxyR (Henderson \& Owen, 1999). It was proposed that OxyR represses transcription by binding to a site located $19 \mathrm{bp}$ downstream from the putative start point for agn43 transcription (Hasman et al., 1999). Three GATC sites, which are targets for methylation by the Dam methylase, are located in the regulatory region of the agn 43 gene (Henderson \& Owen, 1999; Hasman et al., 1999). These sequences are not protected from methylation in an $o x y R$ background and methylation of these sequences abrogates binding by OxyR (Haagmans \& van der Woude, 2000). Thus, the Dam methylase promotes the 'ON' phase, while binding by OxyR has the opposite effect. The oxyR gene, in turn, is regulated by catabolite repression: mutations in crp (encoding cAMP receptor protein, the global regulator of 'catabolite-repressible' genes) or cya (encoding adenylate cyclase) prevent the induction of oxyR expression that occurs in early exponential phase (Gonzalez-Flecha \& Demple, 1997).

We found that introduction of the rpoA341 mutation into cells predominantly in the 'OFF' phase for agn 43 expression results in switching of the population to the ' $\mathrm{ON}$ ' phase as judged by the presence of increased synthesis of Ag43 and an increased efficiency of autoaggregation. Gene fusion analysis suggested that this was due, at least in part, to an increase in transcription of agn43. The rpoA341 mutation results in a single amino acid change (K271E) in the C-terminal domain of the $\alpha$ subunit of RNA polymerase (Thomas \& Glass, 1991). It was previously demonstrated that the stimulation of a number of promoters by different transcriptional activators, including two promoters which require the CRPcAMP complex, is impaired in the rpoA341 mutant (Giffard \& Booth, 1988; Wȩgrzyn et al., 1992; Szalewska-Pałasz et al., 1996; Obuchowski et al., 1997; Gabig et al., 1998), most probably due to a defective interaction between these activators and RNA polymerase. Since efficient expression of oxyR requires the CRP-cAMP complex, one possible reason for the increased transcription of agn43 in the rpoA341 mutant may be a decrease in OxyR synthesis due to a defective interaction between the CRP-cAMP complex and RNA polymerase at the $\operatorname{oxy} R$ promoter. In support of this, bacteria harbouring the $r p o A 341$ allele were found to be sensitive to hydrogen peroxide.

\section{ACKNOWLEDGEMENTS}

We are very grateful to R. Kolter, M. Marinus and G. Storz for providing bacterial strains and to Marta Pasenkiewicz-Gierula for discussions. We thank A. Kuźniacka and M. Ciejka for assistance during some experiments. This work was supported by the Polish State Committee for Scientific Research (project grant 6 P04A 01616 to G. W.) and the Wellcome Trust (grant 050794 to M.S. T.). G. W. also acknowledges financial support from the Foundation for Polish Science (subsidy 14/2000).

\section{REFERENCES}

Arber, W., Enquist, L., Hohn, B., Murray, N. E. \& Murray, K. (1983). Experimental methods for use with lambda. In Lambda II, pp. 433-466. Edited by R. W. Hendrix, J. W. Roberts, F. W. Stahl \& R. A. Weisberg. Cold Spring Harbor, NY: Cold Spring Harbor Laboratory.

Bernstein, C., Bernstein, H., Payne, C. M., Beard, S. E. \& Schneider, J. (1999). Bile salt activation of stress response promoters in Escherichia coli. Curr Microbiol 39, 68-72.

Caffrey, P. \& Owen, P. (1989). Purification and N-terminal sequence of the $\alpha$ subunit of antigen 43 , a unique protein complex associated with the outer membrane of Escherichia coli. J Bacteriol 171, 3634-3640.

Casjens, S. \& Hendrix, R. (1988). Control mechanisms in dsDNA bacteriophage assembly. In The Bacteriophages, pp. 15-91. Edited by R. Calendar. New York \& London: Plenum.

Danese, P. L., Pratt, L. A., Dove, S. L. \& Kolter, R. (2000). The outer membrane protein, antigen 43 , mediates cell-to-cell interactions within Escherichia coli biofilms. Mol Microbiol 37, 424-432.

Diderichsen, B. (1980). $f l u$, a metastable gene controlling surface properties of Escherichia coli. J Bacteriol 141, 858-867.

Drasar, B. S. \& Barrow, P. A. (1985). Intestinal Microbiology: Aspects of Microbiology, vol. 10. Washington, DC: American Society for Microbiology.

Elliott, W. H. (1985). Metabolism of bile salts in liver and extrahepatic tissues. In Sterols and Bile Acids, pp. 303-329. Edited by H. Danielsson \& J. Sjovall. New York: Elsevier.

Gabig, M., Obuchowski, M., Ciesielska, A., Latała, B., Wȩgrzyn, A., Thomas, M. S. \& Węgrzyn, G. (1998). The Escherichia coli 
RNA polymerase $\alpha$ subunit and transcriptional activation by bacteriophage $\lambda$ CII protein. Acta Biochim Pol 45, 271-280.

Giffard, P. M. \& Booth, I. R. (1988). The rpoA341 allele of Escherichia coli specifically impairs the transcription of group of positively regulated operons. Mol Gen Genet 214, 148-152.

Giladi, H., Koby, S., Gottesman, M. E. \& Oppenheim, A. B. (1992). Supercoiling, integration host factor, and a dual promoter system participate in the control of the bacteriophage $\lambda \mathrm{pL}$ promoter. $J$ Mol Biol 224, 937-948.

Goldberg, A. R. \& Howe, M. (1969). New mutations in the $S$ cistron of bacteriophage lambda affecting host cell lysis. Virology 38, 200-202.

Gonzalez-Flecha, B. \& Demple, B. (1997). Transcriptional regulation of the Escherichia coli oxyR gene as a function of cell growth. J Bacteriol 179, 6181-6186.

Haagmans, W. \& van der Woude, M. (2000). Phase variation of Ag43 in Escherichia coli: Dam-dependent methylation abrogates OxyR binding and OxyR-mediated repression of transcription. Mol Microbiol 35, 877-887.

Hancock, R. E. W. (1997). The bacterial outer membrane as a drug barrier. Trends Microbiol 5, 37-42.

Hasman, H., Chakraborty, T. \& Klemm, P. (1999). Antigen-43mediated autoaggregation of Escherichia coli is blocked by fimbriation. J Bacteriol 181, 4834-4841.

Hasman, H., Schembri, M. A. \& Klemm, P. (2000). Antigen 43 and type 1 fimbriae determine colony morphology of Escherichia coli K-12. J Bacteriol 182, 1089-1095.

Henderson, I. R. \& Owen, P. (1999). The major phase-variable outer membrane protein of Escherichia coli structurally resembles the immunoglobulin A1 protease class of exported protein and is regulated by a novel mechanism involving Dam and OxyR. $J$ Bacteriol 181, 2132-2141.

Henderson, I. R., Meehan, M. \& Owen, P. (1997). A novel regulatory mechanism for a novel phase-variable outer membrane protein of Escherichia coli. In Mechanisms in the Pathogenesis of Enteric Diseases: Advances in Experimental Medicine and Biology, vol. 412. Edited by P. S. Paul, D. H. Francis \& D. A. Benfield. New York: Plenum.

Jensen, K. F. (1993). The Escherichia coli 'wild types' W3110 and MG1655 have an $r p h$ frameshift mutation that leads to pyrimidine starvation due to low pyrE expression levels. J Bacteriol 175, 3401-3407.

Kȩdzierska, S., Staniszewska, M., Potrykus, J. \& Wȩgrzyn, G. (1999). The effect of some antibiotic-resistance-conferring plasmids on the removal of the heat-aggregated proteins from Escherichia coli cells. FEMS Microbiol Lett 176, 279-284.

Kjaergaard, K., Schembri, M. A., Ramos, C., Molin, S. \& Klemm, P. (2000a). Antigen 43 facilitates formation of multispecies biofilms. Environ Microbiol 2, 695-702.

Kjaergaard, K., Schembri, M. A., Hasman, H. \& Klemm, P. (2000b). Antigen 43 from Escherichia coli induces inter- and intraspecies cell aggregation and changes in colony morphology of Pseudomonas fluorescens. J Bacteriol 182, 4789-4796.

Kucharczyk, K., Laskowska, E. \& Taylor, A. (1991). Response of Escherichia coli cell membranes to induction of $\lambda$ cl857 prophage by heat shock. Mol Microbiol 5, 2935-2945.
Miller, J. H. (1972). Experiments in Molecular Genetics. Cold Spring Harbor, NY: Cold Spring Harbor Laboratory.

Obuchowski, M., Giladi, H., Koby, S., Szalewska-Pałasz, A., Wȩgrzyn, A., Oppenheim, A. B., Thomas, M. S. \& Wȩgrzyn, G. (1997). Impaired lysogenization of the Escherichia coli rpoA341 mutant by bacteriophage $\lambda$ is due to the inability of CII to act as a transcriptional activator. Mol Gen Genet 254, 304-311.

Owen, P. (1992). The Gram-negative outer membrane: structure, biochemistry and vaccine potential. Biochem Soc Trans 20, 1-6.

Owen, P., Caffrey, P. \& Josefsson, L. G. (1987). Identification and partial characterization of a novel bipartite protein antigen associated with the outer membrane of Escherichia coli. J Bacteriol 169, 3770-3777.

Pope, L. M., Reed, K. E. \& Payne, S. M. (1995). Increased protein secretion and adherence to HeLa cells by Shigella spp. following growth in the presence of bile salts. Infect Immun 63, 3642-3648. Sambrook, J., Fritsch, E. F. \& Maniatis, T. (1989). Molecular Cloning: a Laboratory Manual. Cold Spring Harbor, NY: Cold Spring Harbor Laboratory.

Silhavy, S. J., Berman, M. L. \& Enquist, L. W. (1984). Experiments with Gene Fusions. Cold Spring Harbor, NY: Cold Spring Harbor Laboratory.

Szalewska, A., Wȩgrzyn, G. \& Taylor, K. (1994). Neither absence nor excess of $\lambda \mathrm{O}$ initiator-digesting ClpXP protease affects $\lambda$ plasmid or phage replication in Escherichia coli. Mol Microbiol 13, 469-474.

Szalewska-Pałasz, A., Węgrzyn, A., Obuchowski, M., Pawłowski, R., Bielawski, K., Thomas, M. S. \& Wȩgrzyn, G. (1996). Drastically decreased transcription from CII-activated promoters is responsible for impaired lysogenization of the Escherichia coli rpoA341 mutant by bacteriophage $\lambda$. FEMS Microbiol Lett 144, 21-27.

Thomas, M. S. \& Glass, R. E. (1991). Escherichia coli rpoA mutation which impairs transcription of positively regulated systems. Mol Microbiol 5, 2719-2725.

Warne, S. R., Varley, J. M., Boulnois, G. J. \& Norton, M. G. (1990). Identification and characterization of a gene that controls colony morphology and auto-aggregation in Escherichia coli K12. J Gen Microbiol 136, 455-462.

Wȩgrzyn, G., Glass, R. E. \& Thomas, M. S. (1992). Involvement of the Escherichia coli RNA polymerase $\alpha$ subunit in transcriptional activation by the bacteriophage lambda $\mathrm{CI}$ and $\mathrm{CII}$ proteins. Gene $122,1-7$.

Williams-Smith, H. (1965). Observations on the flora of the alimentary tract of animals and factors its composition. J Pathol Bacteriol 89, 95-122.

Yanisch-Perron, C., Vieira, J. \& Messing, J. (1985). Improved M13 phage cloning vectors and host strain: nucleotide sequence of the M13mp18 and pUC19 vectors. Gene 33, 103-119.

Zheng, M., Doan, B., Schneider, T. D. \& Storz, G. (1999). OxyR and SoxRS regulation of fur. J Bacteriol 181, 4639-4643.

Zilberstein, D., Padan, E. \& Schuldiner, S. (1980). A single locus in Escherichia coli governs growth in alkaline $\mathrm{pH}$ and on carbon sources whose transport is sodium-dependent. FEBS Lett 168, $327-330$.

Received 26 July 2001; revised 22 October 2001; accepted 7 January 2002. 